\title{
Glucose tolerance, insulin sensitivity and adiponectin level in niacin-treated obese rats
}

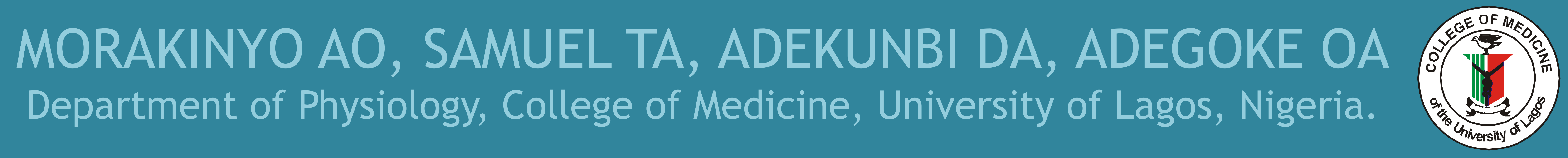

\section{INTRODUCTION}

Obesity is characterized by excessive accumulation or increase in adipose tissue mass (Vazquez-Vela et al., 2008).

Adiponectin secreted by adipose tissue is known to have antiinflammatory, anti-atherogenic and insulin-sensitizing properties.

Niacin is an old lipid-modifying drug that has favourable effects on all traditionally measured lipid parameters (Song \& FitzGerald 2013).

Animal studies have shown that niacin stimulates adiponectin secretion, and, result in increased adiponectin mRNA (Plaisance et al., 2009).

Previous reports indicate that niacin has negative effects on glucose control and insulin metabolism in hyperlipidaemic or non-obese subjects. However, there is no known report of its effects on glucose and insulin sensitivity under obese condition.

The present study was therefore carried out to investigate the effect of niacin on adiposity, glucose tolerance, insulin sensitivity and adiponectin level in HFD-induced obese rats.

\section{METHODS}

\section{Grp 1: Normal diet for 14 weeks}

Grp2: HFD diet for 14 weeks

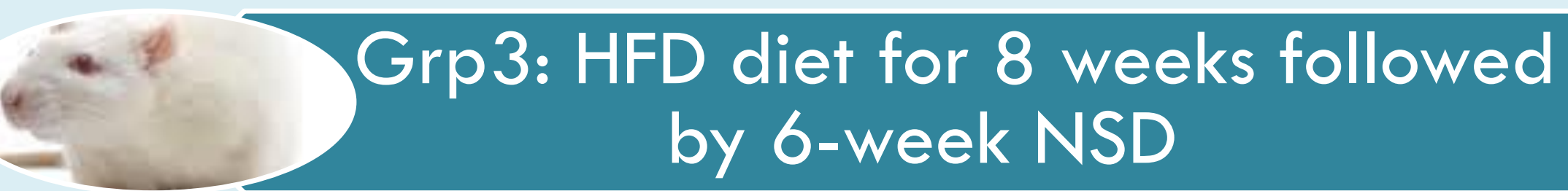

\section{by 6 -week NSD}

Water and food were available ad libitum to the rats throughout the experimental period.

Glucose tolerance test (GTT) and insulin tolerance test (ITT) performed in fasted condition; area under the curve (AUC) was thereafter calculated.

Triglyceride (TG), Cholesterol (CHOL), High Density Lipoprotein (HDL) and Low Density Lipoprotein (LDL) were determined with an automatic blood chemical analyser (BT 2000 Plus, Germany).

Visceral fat (epididymal, omental and retroperitoneal fat deposits were isolated and weighed (Kim et al., 1999).

Serum insulin and adiponectin were assayed using the ELISA kits (Elabscience, Wuhan, China).

Data shown as mean \pm SEM, compared by ANOVA followed by SNK's multiple comparison test. ${ }^{*} P<0.05$ vs. ND; ${ }^{* *} P<0.01$ vs. ND; $\# P<$ 0.05 vs. HSD, \#P<0.01 vs. HSD.

${ }^{\#} H F D=$ high fat diet, NSD $=1 \mathrm{mg} / \mathrm{kg}$ niacin-supplemented diet

\section{RESULTS}

\begin{tabular}{ccccc}
\hline Parameters & Timeline & Control & Obese & Obese+Niacin \\
\hline Body & Baseline & $51.00 \pm 1.32$ & $52.00 \pm 1.23$ & $49.14 \pm 1.53$ \\
Weight (g) & $4 \mathrm{wk}$ & $86.14 \pm 4.72$ & $115.88 \pm 3.88^{*}$ & $111.42 \pm 8.51^{*}$ \\
& $8 \mathrm{wk}$ & $131.14 \pm 5.91$ & $176.29 \pm 5.26^{*}$ & $166.4 \pm 9.61^{*}$ \\
& $14 \mathrm{wk}$ & $194.42 \pm 4.64$ & $273.33 \pm 4.47^{*}$ & $212.4 \pm 8.02^{\#}$ \\
\hline Food & Baseline & $62.57 \pm 3.24$ & $71.14 \pm 1.86$ & $72.14 \pm 4.50$ \\
Intake (g) & $4 \mathrm{wk}$ & $90.71 \pm 3.63$ & $94.29 \pm 2.10$ & $96.57 \pm 2.99$ \\
& $8 \mathrm{wk}$ & $100.43 \pm 2.27$ & $104.71 \pm 2.76$ & $107.57 \pm 3.79$ \\
& $14 \mathrm{wk}$ & $122.86 \pm 2.14$ & $122.86 \pm 2.64$ & $128.43 \pm 3.73$ \\
\hline \% Weight & $4 \mathrm{wk}$ & 68.9 & 122.84 & 126.74 \\
Gain & $8 \mathrm{wk}$ & 157.13 & 239.01 & 238.62 \\
& $14 \mathrm{wk}$ & 281.21 & 425.63 & 332.23
\end{tabular}

Body weight, weight gain and food intake in control and experimental rats.
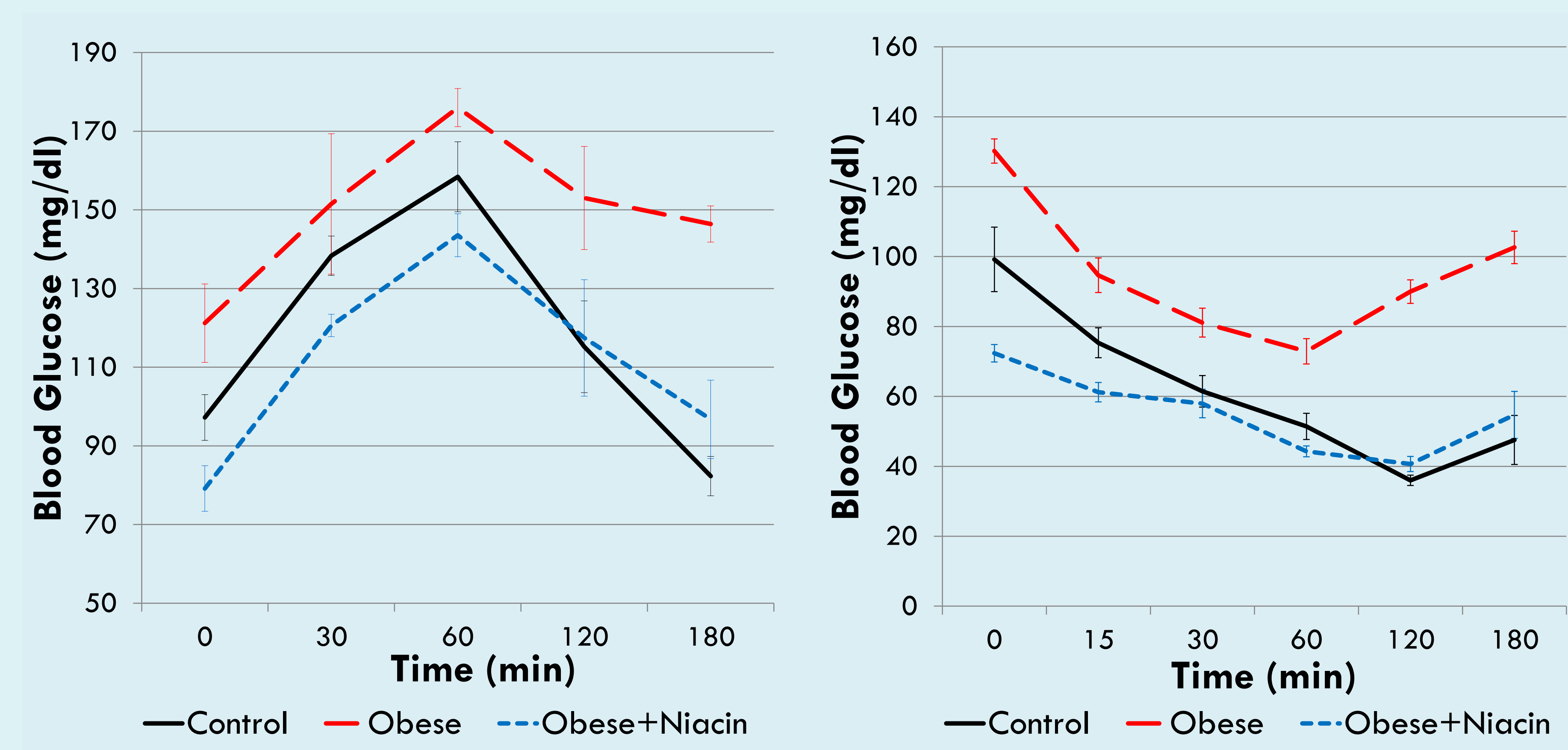

Glucose response curves during an GTT \& ITT in control and experimental rats

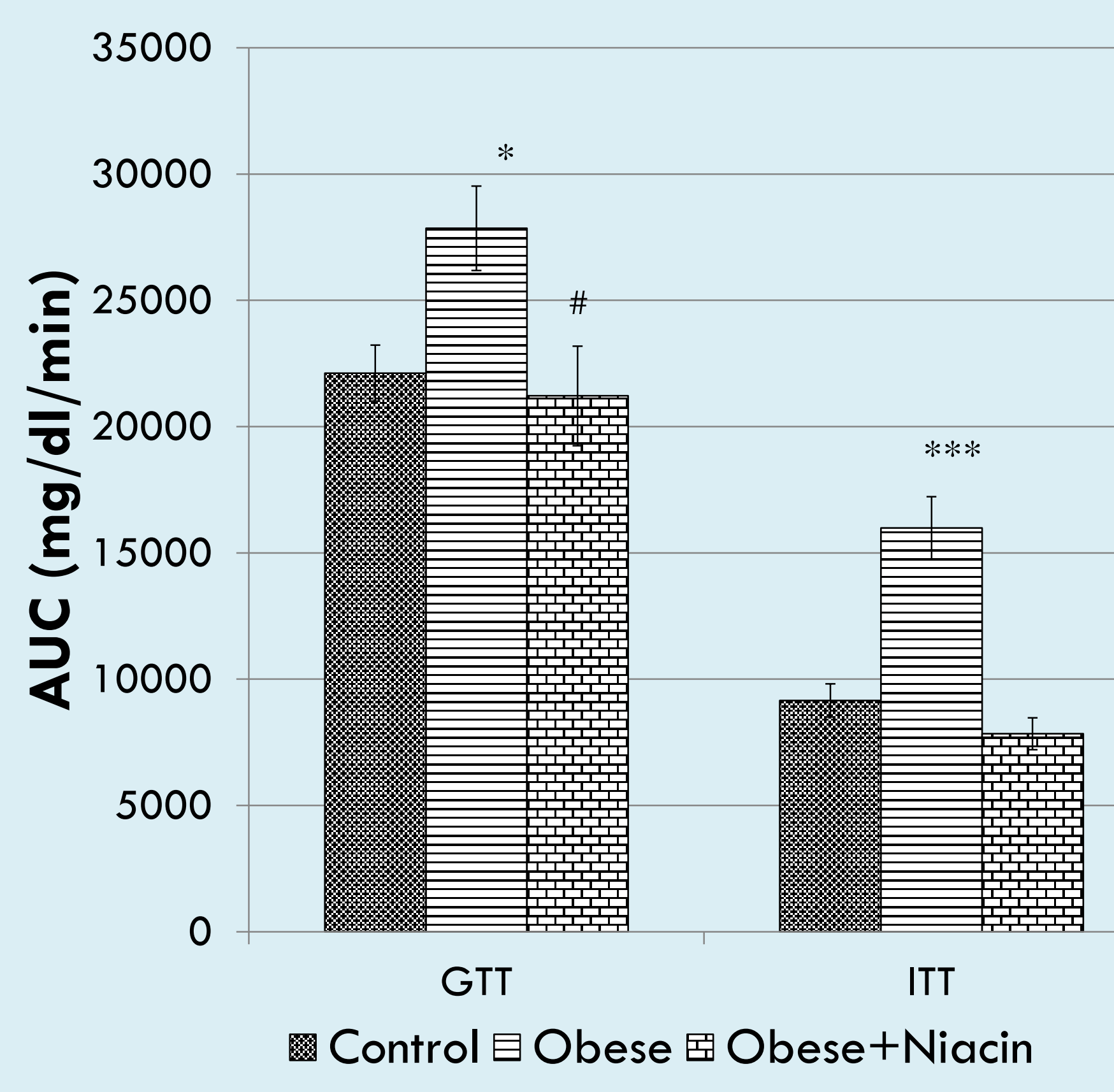

AUC for GTT \& ITT in rats.

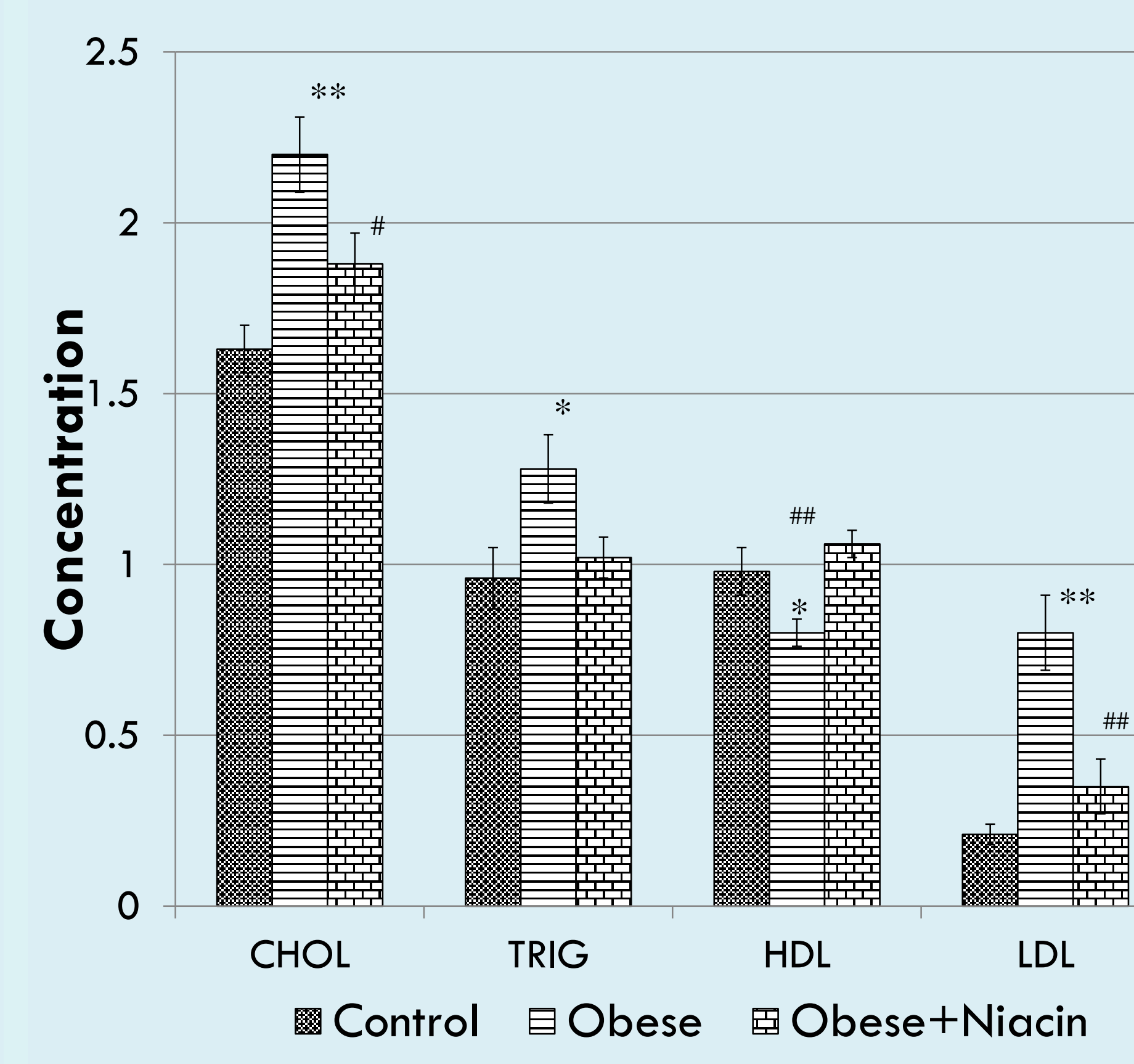

Concentration of plasma lipids in rats.

\begin{tabular}{|c|c|c|c|}
\hline Parameters & Control & Obese & Obese+Niacin \\
\hline Epididymal fat pad (g) & $0.93 \pm 0.04$ & $1.15 \pm 0.05^{*}$ & $1.01 \pm 0.07$ \\
\hline Epididymal fat pad / BW ratio & 0.48 & 0.38 & 0.48 \\
\hline Omental fat pad (g) & $0.85 \pm 0.06$ & $1.18 \pm 0.05^{* *}$ & $0.95 \pm 0.05^{\# \#}$ \\
\hline Omental fat pad / BW ratio & 0.44 & 0.4 & 0.46 \\
\hline Retroperitoneal fat pad (g) & $0.98 \pm 0.06$ & $1.29 \pm 0.09 *$ & $1.09 \pm 0.09$ \\
\hline Retroperitoneal fat pad / BW ratio & 0.5 & 0.47 & 0.51 \\
\hline
\end{tabular}

Effect of niacin supplementation on visceral fat in rats.

\begin{tabular}{lccc}
\hline Parameters & Control & Obese & Obese+Niacin \\
\hline Insulin (pmol/l) & $693 \pm 35$ & $1076 \pm 98^{* *}$ & $842 \pm 64^{\#}$ \\
Adiponectin $(\mathrm{ng} / \mathrm{ml})$ & $986 \pm 87$ & $651 \pm 58^{* *}$ & $813 \pm 72^{\# \#}$ \\
\hline
\end{tabular}

Effect of niacin supplementation on insulin and adiponectin levels in rats.

\section{KEY FINDINGS}

- The body weight and weight gain of niacin-supplemented rats were markedly lower when compared with HFD-fed obese rats.

- Niacin-supplemented rats had improved time-course glucose control and insulin sensitivity demonstrated by a significantly lower AUC values for glucose curves.

- The plasma levels of cholesterol, triglycerides and low-density lipoprotein in niacin-supplemented rats were within values of control rats.

- Niacin appears to exert beneficial effect on adiposity, plasma lipids, insulin sensitivity, glucose tolerance, and specifically modulates the level of adiponectin under obese condition in rats.

\section{REFERENCES}

Kim YW et al (1999) J Korean Med Sci 14; 539-45

Plaisance et al (2009) Am J Physiol Endocrinol Metab 296; E549-58.

Vazquez-Vela et al (2008) Arch Med Res 39; 715-28.

Song WL, FitzGerald GA. (2013). J Lipid Res 54;2586-94.

The Physiological Society
We thank Sunday Ogunowo for the technical assistance provided. 